\title{
An Efficient Neural Network Model for the Identification of Stress using Electrocardiogram
}

\author{
Mithun H R, Suchitra M
}

\begin{abstract}
People are facing numerous pressures in their daily routine in the latest society. Stress has traditionally has been described as action from a calm state to an emotional state in order to preserve the integrity of organism. Stress observation is very important for mental wellbeing and early identification of stress related disorders. Stress is to learn the body response in stressful state, whenever the body reaction is activated that means the heart rate and blood pressure will raise and several hormones enter our bloodshed. These hormones and bodily changes may increases our performances to a particular extent. Everyone's response to stress is discreet, and not all stress is bad. Someone may discover a significant condition of pressure to be enjoyable, while others may find it stressful. However, individuals also have different stress symptoms. stress area can also recognize using frequency and excitation of a speech signal, Since the biomedical signals are consistently related to central nervous system, therefore physiological parameters are the best way to understand the human emotions.
\end{abstract}

The present work is focused on stress identification from Electrocardiogram using ECG physiologic net database, then entire environment of ECG signal characteristics i.e. mean heart rate variability $(H R V)$, standard deviation of all $R-R$ interval (SDNN), square root mean of the sum of the square difference between $R-R$ interval (RMSSD) and number of consecutive $R-R$ interval variations greater than 50ms (NN50), these features are extracted using Pan-Tompkins algorithm, then it is trained and validated to machine learning using back-propagation algorithm in neural network model. With the help of these features (mean HRV, SDNN, RMSSD and NN50), the study can be analyzed whether a person is under stress or not. Thus how the suggested technique provides the subjective information which helps the doctor to find out whether the person is under stress or not.

Keywords- Human emotions, Physiological signals, Pan-Tompkins algorithm and Neural Network.

\section{INTRODUCTION}

People are facing numerous pressure in their daily routine living in the latest society. Stress has traditionally been described as action from a calm state to an emotional state in order to preserve the organism's integrity [1]. Stress is considered in the psychobiological level as a sequence of psychological, cognitive and behavioral complications regulated by the human central nervous system (CNS) and the

Revised Manuscript Received on July 22, 2019.

Mithun H R, M.Tech Student: Dept. ECE, Vidyavardhaka college of Engineering, Mysuru, India. Mail id: mithunhrm07@gmail.com

Dr.Suchitra M, Assoc Prof and PG-Coordinator: Dept.ECE, Vidyavardhaka college of Engineering, Mysuru, India. Mail id: suchitram.suchi@gmail.com

peripheral nervous system (PNS). [2]. The favorable stress is usually lucrative for the topics and does not require them to trade with complicated problems or to adapt to the latest scenario. If stress hurts the topics and can cause side effects such as mental and physical issues, this stress is regarded to be negative stress. Usually, it's the adverse stress when we point out the "stress" in general terms. It has been discovered that if the stress acquired from family or working environment continues to appear, it will have serious effects on everyone [3].

The researcher found that chronic stress can lead to different mental and physical problems, including cardiovascular disease and immune deficiency. [4]. The researcher later discovered that physiological reactions, facial characteristics (facial expressions, eye gaze and eye blinks) and voice could be the channels for studying the emotional state of the person. [5]. Thus, these body expressions ' features were examined. Usually, physiological responses were investigated by estimating the characteristics of an individual's physiological signals such as electrocardiography (ECG), electromyography (EMG) and electrodermal activity (EDA) under various affective states [6]. The facial characteristics were generally examined by estimating characteristics such as facial expressions, eye motions and dilation from facial pictures or videos collected by sensors such as cameras. The voice was evaluated by estimating vocal features such as loudness and basic speech frequency. Meanwhile, the potential for adopting stress identification expressions of the body was examined and some detection techniques were suggested. Although it was discovered that using body expressions such as physiological signals to define an individual's stress state is not an simple task, nor is it a direct task. No wonderful rules have been discovered and validated. The cope with the likelihood and interest of recognizing stress from heterogeneous information and suggest an approach to the identification method.

Nevertheless, paper spare is structured as follows: work linked in chapter II, current stress identification in pre-processing, extraction characteristics, measures of resemblance, and classification. In Section III, the suggested framework and classification algorithm are explained in detail. We represent with debate on the experimental outcomes in chapter IV. Finally, we conclude the job with future improvements in Section V. 


\section{RELATED WORK}

Total performance of relative characteristics is found in[ 7]. Rearrangement takes place using six classifier types: 1) Support vector machine with linear kernel 2) SVM with radial base function kernel 3) k-nearest neighbor (k=1-4) 4) Principal component analysis and SVM with linear kernel 5) PCA and SVM with RBF kernel 6) PCA and neighbor closest. In[ 8], with the advantage of bio-sensors, Smart Mobile Health Monitoring System obtains the physiological information of humans. The information is collected in the internet of the sensor and a compact of the information obtained is sent to the personal computer of a patient. These devices transmit data to the medical server for determination. After the data is analysed, the medical server send response to the patient's private computer. Depending on the response, patients can take the necessary measures. The IMHMS consists of three parts. They are wearable network of Body Sensors ; Private Home Server and Smart Medical Server for patients. This sensor network is created in the body of the patient using wearable bio-sensors. The collection of significant information is carried out with the assistance of wearable sensor detectors.

In [ 9 ], a heart rate monitor (HRM) was used to define mentally stressful event. The method is linked to the main condition of dynamics, after inquiry of the variation of the heart rate. In [10], the Galvanic Skin Response (GSR) is a skin conductivity measurement that is significantly linked to human emotional condition during stress and activation stage. Skin behavior level or reaction (SCL and SCR) is a way to estimate human skin's electrical behavior that varies with human mental and emotional state. Two electrodes are positioned so that the conductive pathway between them crosses the hand palm for the research of galvanic skin response. Skin resistance or reciprocity; this parameter is used for the calculation of skin conductance. Ohm's Law says that the strength of the skin is equal to the voltage applied by the current passed through the skin between two electrodes on the skin. Thus, the prospective skin difference is calculated and accomplished through the data acquisition system. In[11], they discover a fresh spectral feature that estimates the equilibrium measure of the self-sustaining nervous system by combining data from the respiration task's power spectral density, i.e. breathing and variability in heart rate.

They also evaluate the characteristics such as mean, median and standard deviation from the rate and reaction of skin conductivity. Using the logistic recession pattern, they evaluate outpatient stress In [ 12 ], indicated principle machine learning mode to classify big information of continually acquired, multivariable physiological information, using wearable patient monitors, also provides early warning of serious physiological issue, so that a degree of estimation care can be given. The article examined the literature produced on wearable sensors and devices for tracking different human actions in [13]. It reveals that there will be many more lightweight, high-performance wearable devices available to monitor a wide variety of actions
An instance could be the work of Cinaz et al.[14 ], who regarded a 3 classification problem to divide the mental job quantity of the office worker into low, medium and high groups using only an ECG signal and HRV (eight time domain characteristics and LF / HF ratio) to achieve right predictions for 6 out of 7 topics using Linear Discriminant Analysis (LDA) [ 15 ] Classified. Wijsman and others. [ 16 ] ECG evaluated along with trapezoid muscle conductivity, respiration and EMG. They accomplished an precision of 80 percent and 69.1 percent in non-stress and stress detection, using a quite decreased set of characteristics (only five characteristics) obtained from those signals, including Heart Rate. HRV, ECG, EMG, EDA and ST were evaluated in the research conducted by Palanisamy et al.[17 ] and a total of 148 characteristics were acquired. Instead of establishing a classification scheme based on the entire set of signals, classification accuracy of each of the signals was evaluated. Results show that ECG and HRV performed well in the detection of stress relative to the other signals. Precisely, the highest classification rate for HRV was $93.75 \%$, followed by ECG $76.25 \%$ and the minimum classification rate for EDA signals was $70.83 \%$. Melillo and others. [18] In contrast to most State of the Art works that tested them in laboratory settings, the effect of stress on HRV parameters was evaluated in real-life conditions. To estimate the ECGs of learners, they chosen two critical moments: during an oral examination and after holidays. They used 13 non-linear HRV and LDA characteristics to classify stressed and relaxed conditions

\section{PROPOSED WORK}

\section{A. Problem description}

Stress is fairly strong, it can change their activity and put them at danger for individuals who are always facing pressing circumstances (such as a fireman or astronaut). Therefore, measuring employee stress and then providing feedback solving to regulate this state is quite important. Stress surveillance is therefore essential for mental well-being and early detection of associated illnesses, the present research focuses on identifying stress from ECG signals using back-propagation algorithms in neural networks

\section{B. System architecture}

The system architecture in Figure 2 demonstrates the following elements: Pre-processing, Feature Extraction, Training, Classification and Testing

\section{1) Pre-processing}

Pre-processing is our suggested system's original method. Noise is heavily influenced by the ECG signal, and the signal is very weak. The primary digital signal issue is interference like noisy signals from the $50 \mathrm{~Hz}$ frequency power supply as well as inhalation muscle interference. Before the signal is used in the stress detection phase, this interference such as loud signal should be removed, so notch filter is used for fixed 
frequency noise source such as line frequency (50 or $60 \mathrm{HZ}$ ) noise on the command. Then it was used for the extraction of the function after removing the interference such as noise

\section{2) Feature extraction}

This phase characterizes the perspectives on the situation of extraction of features. It is said that features are characteristics that represent all the biological signals. Using the Pan and Tompkins algorithm, the QRS complexes are identified where a digital band pass filter is performed. It can decrease the fake identification induced by the various kinds of interferences in the ECG signal By matching the ordinary QRS complex range. The pass band that increases QRS power is within the range of $5 \mathrm{HZ}-35 \mathrm{HZ}$. The derivative method stops the low-frequency elements of $\mathrm{P}$ and $\mathrm{T}$ waves and gives the gain to high-frequency elements resulting from the high slopes of the QRS complex, then the square waveform passes through the motion window integrator, squaring the signal patterns from point to point in nonlinear transformation.

\section{3) Train to Machine learning}

They train to SVM after extracting the features because it's a basic classifier, but it can't correctly classify because the distinction in patient stress and not stress information is very minute, then train to the neural network using back propagation algorithm. The weights of the inputs are adapted with supervised learning to train back propagation. In the teaching strategy of neural networks, the training set's input values are rated with the necessary output. In the past, the initialization of weight is performed at random speed. Each training dataset provides the perceptron. By comparing the perceptron output with each input dataset, the necessary output is acquired.

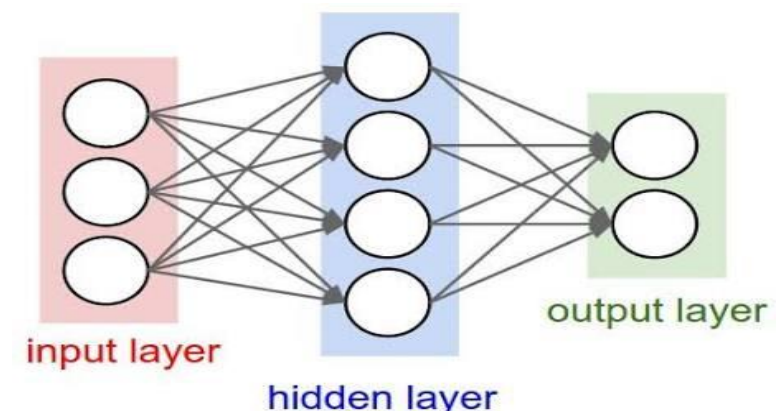

Figure-1: Neural network architecture

\section{A. Back propagation algorithm}

The back propagation algorithm is now widely used to train neural networks. The scheme operates by estimating the non-linear relationship between input and output values internally by tuning weights on the connector.

Algorithm: Back propagation

Input: Data setting for the sample of training and appropriate destination outputs.

Output: Trained neural network.

Enter bias and weights to tiny random numbers

// Sending input vector:

for every neuron a in input layer,

$O_{\mathrm{A}}=I_{\mathrm{a}}$

for every neuron a in the hidden and output layer,

$I_{\mathrm{a}}=\sum_{\mathrm{b}} W_{\mathrm{ab}} O_{\mathrm{b}}+\theta_{\mathrm{a}}$

$O_{\mathrm{A}}=1 / 1+e^{-I^{\mathrm{a}}}$

// Propagating error back to front:

for every neuron a in output layer,

$\operatorname{Error}_{\mathrm{a}}=O_{\mathrm{a}}\left(1-O_{\mathrm{a}}\right)\left(T_{\mathrm{a}}-O_{\mathrm{a}}\right)$

for every neuron a in hidden layer,

Error $_{\mathrm{a}}=O_{\mathrm{a}}\left(1-O_{\mathrm{a}}\right)\left(\sum_{\mathrm{m}}\right.$ Error $\left._{\mathrm{m}} W_{\mathrm{am}}\right)$

for weight linked with every edge in the network,

$\Delta W_{\mathrm{ab}}=(1) \operatorname{Error}_{\mathrm{a}} O_{\mathrm{b}}$

$W_{\mathrm{ab}}=W_{\mathrm{ab}}+\Delta W_{\mathrm{ab}}$

for rest of bias $\theta_{\mathrm{a}}$ in network,

$\Delta \theta_{\mathrm{a}}=$ (1) Error $_{\mathrm{a}}$

$\theta_{\mathrm{a}}=\theta_{\mathrm{a}}+\Delta \theta_{\mathrm{a}}$

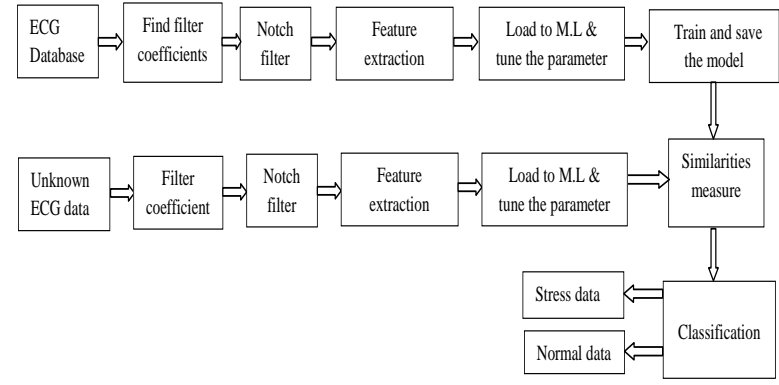

Figure-2: System architecture.

\section{4) Testing strategy}

The approach used for testing is described below:

a) Features to be tested - The features to be tested, most importantly include the estimation of Mean HRV, SDNN, RMSSD, NN50 of an individual patient for the proper execution of the entire model.

b) Items to be tested - The unknown ECG data to be tested with include all the features of individual patient, which together form the whole model.

c) Purpose of testing - The purpose of the testing is to check whether a patient is under stress or not is the main source of the project. 


\section{RESULTS AND DISCUSSION}

The model has been taken from the training database, the test information is fed to the model, then the test data generates the test information result to calculate the outlook effectiveness and then result as if the individual is suffering from stress or normal.

Experiments are performed for the stress

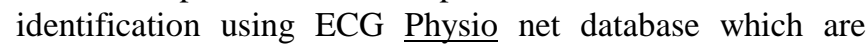
trained by the neural network using back propagation algorithm in Mat lab software. The gain results are represented by the graphical user inter phase. Thus how it helps to classify, if a person is under stress or not, For mental well-being and early detection of associated illnesses, this is crucial. The simulation below provides the stress plot and the normal person.

\section{A) Plot for Stress data}

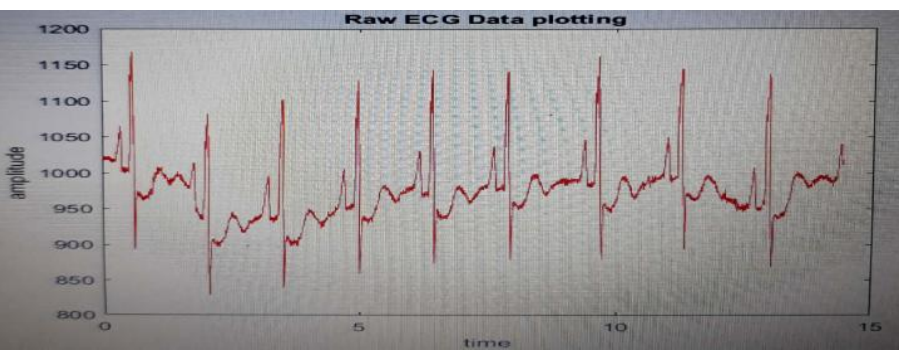

Figure-3: Gives the plot which intern gives the performance graph of ECG raw data signals of a stress person.

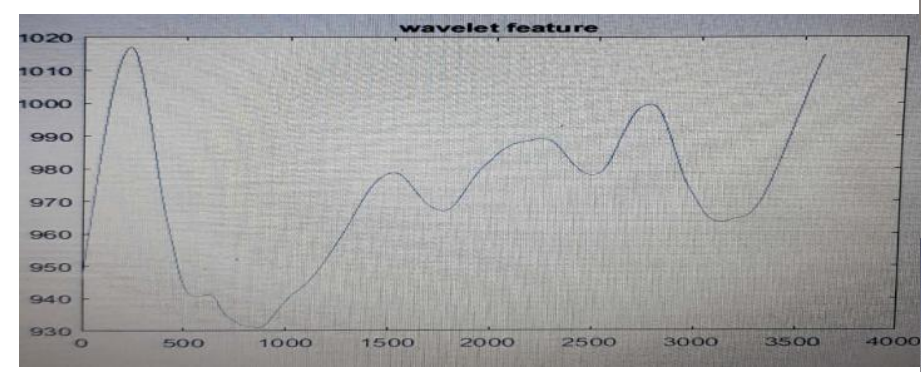

Figure-4: Here discrete wavelet transform is applied to raw ECG data to calculate the statistical parameters of wavelet coefficients such as mean, median and standard deviation of stress person.

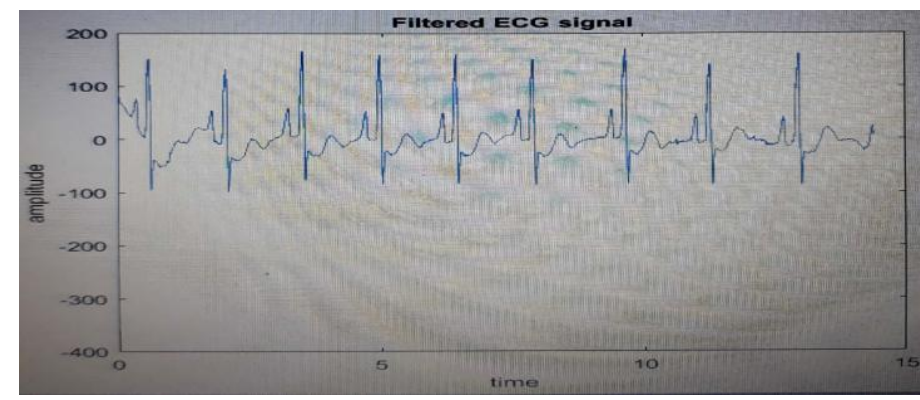

Figure-5: Here notch filter is applied to ECG raw data to remove the interference present in it. The plot shows the filtered ECG signal of a stress person

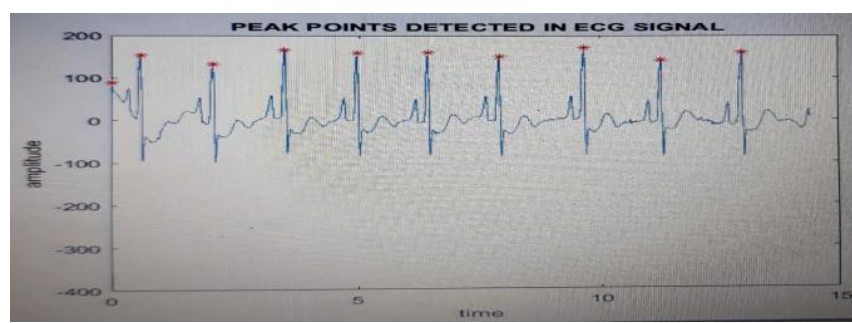

Figure-6: The plot shows the peak point detection in the filtered ECG signal of a stress person

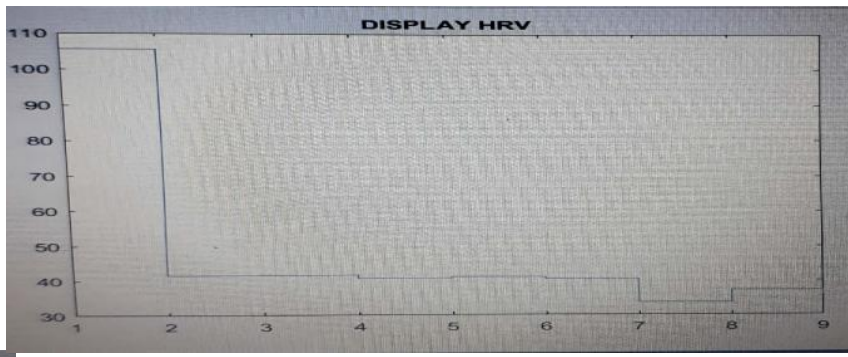

Figure-7: The plot gives the heart rate variability of a stress person

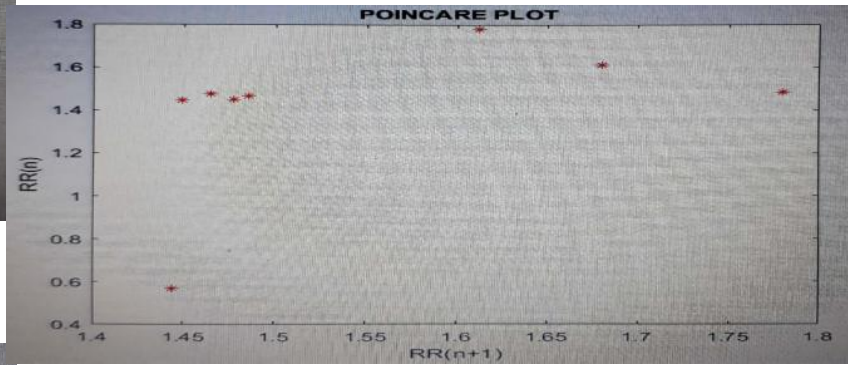

Figure-8: Poincare plot which produces the information regarding entire R-R intervals

B) Plot for Normal data

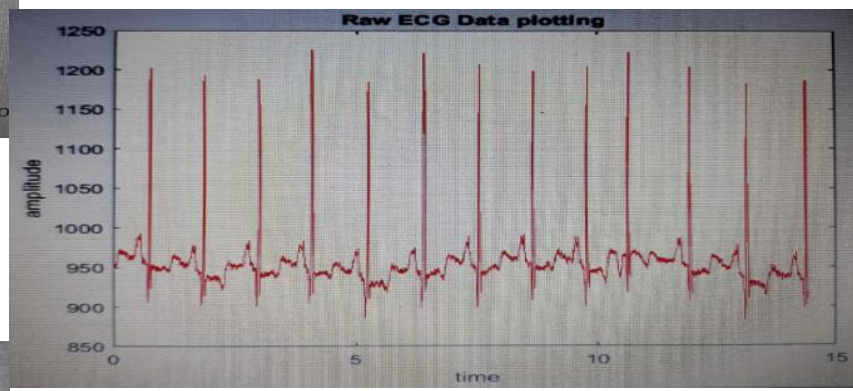

Figure-9: Gives the plot which intern gives the performance graph of ECG raw data signals of a normal person. 


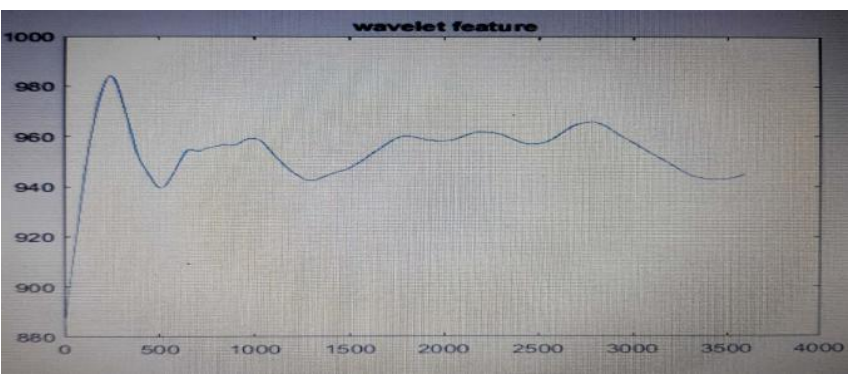

Figure-10: Here discrete wavelet transform is applied to raw ECG data to calculate the statistical parameters of wavelet coefficients such as mean, median and standard deviation of normal person.

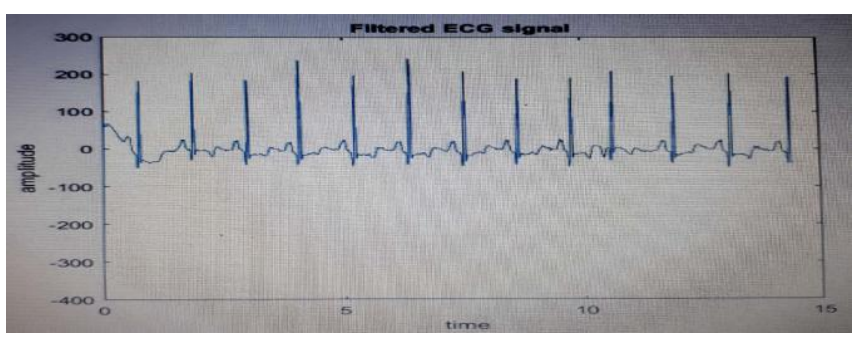

Figure-11: The notch filter here is used to remove the interference current in ECG raw information. The plot demonstrates the filtered ECG signal of an normal person.

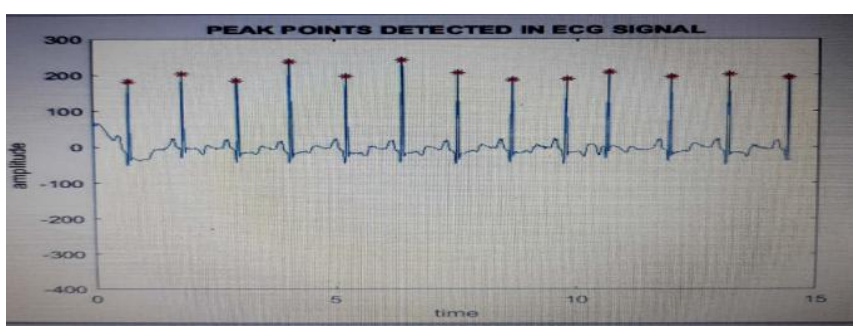

Figure-12: The plot indicates a normal person's maximum detection point in the filtered ECG signal

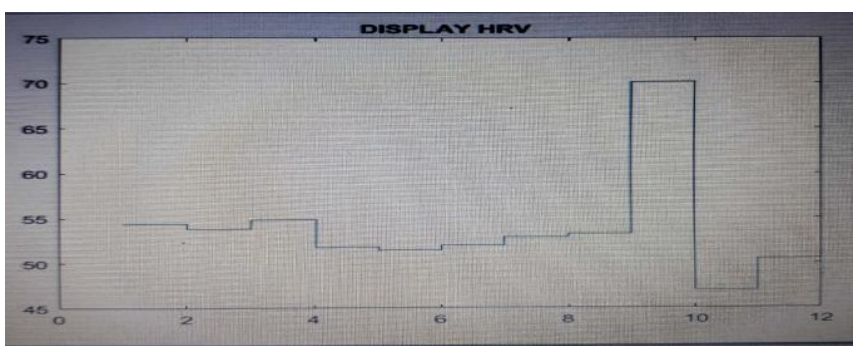

Figure-13: The plot shows a normal person's heart rate variability

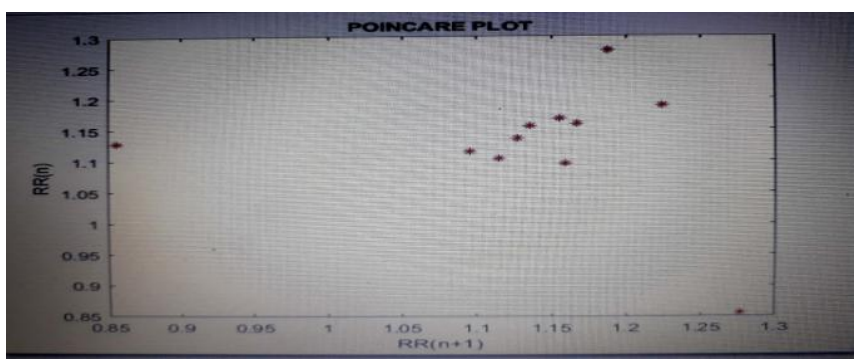

Figure-14: demonstrates the Poin care plot that generates data about a normal person's complete R-R intervals.

\section{CONCLUSIONS}

In this job, we suggest to bundle the training set region into standardized groups on the grounds of self-reported feelings and train and test the regions of the same group to construct the pattern to distinguish the nature of that stimulus, so how the model is created to know from the training information set and is tested with another set of information for the test stage with assisted vector machine and neural network classifier model for identification of stress. This study can be analysed whether a person is under stress or not. The proposed method gives subjective information which helps Doctor to find out if a person is suffering from stress or not with help of ECG signals.

Future work should focused on stress level identification of patient.Because stress is very high for the people who will face the urgent situation,It can change their activity and put them in difficulty, so it is very essential to calculate an individual's stress level and to provide them with a solution because it is very essential for ordinary people and to detect stress levels early.

\section{REFERENCES}

[1] Jennifer A Healey and Rosalind W Picard. Detecting stress during real-world driving tasks using

physiological sensors. Intelligent Transportation Systems, IEEE Transactions on, 6(2):156-166, 2005.

[2] H SELYE. The stress of life. New York: McGraw-Hill, 1956.

[3] Tarani Chandola, Eric Brunner, and Michael Marmot. Chronic stress at work and the metabolic syndrome: prospective study. Bmj, 332(7540):521-525, 2017.

[4] Andreas Riener, Alois Ferscha, and Mohamed Aly. Heart on the road Hrv analysis for monitoringa driver's affective state. In Proceedings of the 1st International Conference on Automotive User Interfaces and Interactive Vehicular Applications, pages 99-106. ACM, 2009.

[5] David F Dinges, Robert L Rider, Jillian Dorrian, Eleanor L McGlinchey, Naomi L Rogers, Ziga Cizman, Siome K Goldenstein, Christian Vogler, Sundara Venkataraman, and Dimitris N Metaxas. Optical computer recognition of facial expressions associated with stress induced by performance demands. Aviation, space, and environmental medicine, 76(6):B172-B182, 2015.

[6] Leon JM Rothkrantz, Pascal Wiggers, Jan-Willem A van Wees, and Robert J van Vark. Voice stressanalysis. In International conference on text, speech and dialogue, pages 449-456.Springer,2014.

[7] Akane Sano and Rosalind W. Picard "Stress Recognition using Wearable Sensors and Mobile Phones" 2013 IEEE DOI 10.1109/ACII.2013.117.

[8] Rifat Shahriyar1, Md. Faizul Bari2, Gourab Kundu3, Sheikh Iqbal Ahamed4, and Md. Mostofa"Intelligent Mobile Health Monitoring System (IMHMS)" International Journal of Control and Automation Vol.2, No.3, September 2009.

[9] Jongyoon Choi and Ricardo Gutierrez-Osuna" Using Heart Rate Monitors to Detect Mental Stress " 2009 IEEE Body Sensor Networks, DOI 10.1109/P3644.12.

[10] Sankhadip Saha, Papri Nag, Mrityunjay Kr. Ray "A Complete Virtual Instrument for Measuring and Analyzing Human Stress in Real Time "2014 IEEE International Conference on Control, Instrumentation, Energy Communication(CIEC).

[11] Jongyoon Choi, Been Ahmed, Ricardo GutierrezOsuna Development and Evaluation of an Ambulatory Stress Monitor Based on Wearable Sensors IEEE Transactions On Information Technology In Biomedicine, Vol. 16, No. 2, March 2012.

[12] L. Clifton, D.A. Clifton, Marco A. F. Pimentel, J. Peter, Predictive monitoring of mobile patients by combining clinical observations with data from wearable sensors IEEE journal of biomedical and health informatics, vol. 18 , no. 3 , may 2014. 
[13] Subhas Chandra Mukhopadhyay, Fellow Wearable Sensors for Human Activity Monitoring: A Review IEEE Sensors Journal,Vol.15,No.3,March2015.

[14]B. Cinaz, B. Arnrich, R. La Marca, G. Tröster Monitoring of mental workload levels during an everyday life office-work scenario Personal Ubiquitous Comput., 17 (2) (2013), pp. 229-239,

[15]S. Mika, G. Ratsch, J. Weston, B. Scholkopf, K. Mullers, Fisher discriminant analysis with kernels, in: Neural Networks for Signal Processing IX: Proceedings of the 1999 IEEE Signal Processing Society Workshop (Cat. No. 98TH8468),

[16] J. Wijsman, B. Grundlehner, H. Liu, J. Penders, H. Hermens Wearable physiological sensors reflect mental stress state in office-like situations 2013 Humaine Association Conference on Affective Computing and Intelligent Interaction, IEEE(2013),pp. 600605

[17] K. Palanisamy, M. Murugappan, S. YaacobMultiple physiological signal-based human stress identification using non-linear classifiers Electron. Electr. Eng., 19 (7) (2013), pp. 80-85 [18] P. Melillo, M. Bracale, L. PecchiaNonlinear heart rate variability features for real-life stress detection. Case study: students under stress due to university examination 